\title{
Palm Vein Recognition Using Directional Features Derived from Local Binary Patterns
}

\author{
Wenjing $\mathrm{Lu}^{\mathrm{a}^{*}}$, Meini $\mathrm{Li}^{\mathrm{a}}$ and Lei Zhang ${ }^{\mathrm{b}}$ \\ ${ }^{a}$ School of Technology, Harbin University, Harbin, China \\ ${ }^{\mathrm{b}}$ School of Art and Design, Harbin University, Haring, China \\ luwj116@outlook.com,limn@hrbu.edu.cn, larrycheung@163.com
}

\begin{abstract}
Vein-based biometrics is a newly developed technology for personal recognition, and it is widely used in practice and intensively studied. This paper proposes a method for palm vein recognition based on the directional information derived from local binary patters. In the proposed method, palm vein images are firstly enhanced using a multi-scale Gaussian matched filter to emphasize vein patterns before feature extraction. After that, local binary patterns are extracted from the enhanced palm vein images. Considering that the direction is the most discriminative feature of veins, the directional information is then computed from the local binary patterns. The computed palm vein features are represented as binary series, therefore, similarities can be computed efficiently by binary operation. Experiments carried out over the near infer-red band of the PolyU multispectral database shows the superiority of the proposed method on verification accuracy to some state-of-the-art literatures.
\end{abstract}

Keywords: palm vein, Gaussian matched filter, local binary pattern, directional information

\section{Introduction}

Biometrics, which uses human physical or behavioral traits for personal identification, is a prominent technology for security. For the past few decades, biometric technology has gained great improvement.

Among all the traits which are frequently used for personal recognition, physical traits from human hand, including fingerprint [1- 2], palmprint [3-4], hand geometry [5-6], palm vein [7-9], and finger vein [10], etc, attracted particular interest, because traits from hands are easier to acquire by acquisition devices and can provide reasonable accuracies. These two characteristics of hand traits make them very competent for establishing practical biometric systems.

Using vein features from hand is a new trend in recent biometric researches. Vein features which can be used for personal recognition include palm vein, dorsal hand vein, and finger vein. The chemical property of blood in vein makes it absorbs more infer-red lights than the tissues around it, and thus vein structures can be clearly captured under infer-red illuminations in either reflective way or transmit way by an infer-red sensed image sensor. This is the basic principle of vein photography. Besides the property of hand traits mentioned above, the most distinguishing advantage of vein features is that they are difficult to fake, because veins lie under human skin, and can hardly be seen in visual light, so it is difficult to fake a person's vein features in biometric systems.

Because of their particular advantages, hand veins are usually used for establishing biometric systems. From the literatures, hand vein recognition methods fall into one of the following two categories, i.e., structure-based ones and texture-based ones.

${ }^{*}$ Corresponding Author 
Structure-based methods are based on the structural characteristics of vein patterns, which are stable throughout human life. Hence, it is believed that structure-based features are more robust to the variances of imaging conditions than texture-based features. Structural features that can be extracted from vein patterns include points, curve- or linelike features, structure of vein nets, etc. Zhang [11] used Gaussian matched filter to convolve the palm vein image, and obtained vein structure information by binarizing the response image. Huang [12] extracted finger vein by using a wide line detection operator. Wang [13] proposed a dorsal hand vein recognition method based on cross points and ending points extracted from vein skeleton. Miura [14] investigated finger vein recognition by using the repeated line tracking algorithm. Another advantage of structurebased methods lies on that the structural features are relatively stable over the variances of the quality of palm vein images. However, in structure-based methods, vein images preprocessing is usually needed to get the vein skeletons, and therefore, the accuracy of such methods may be affected by the performance of preprocessing algorithms. Besides, since only the structure information is used as features, and other discriminative information is ignored, the accuracies of structure-based methods are usually not so high as those of texture-based methods.

Compared with structure-based methods, the idea of texture-based methods are more straightforward. They consider vein patterns as textures, and vein recognition as texture classification issues. In such methods, texture feature extraction algorithms are used for representing vein features. Han [15] used Gabor filters to convolve with vein images for extracting vein features, and then a nearest neighbor classifier was employed for recognition. Yang [16] extracted vein features by using the energy values of the responses of steerable filters. $\mathrm{Bu}$ [17] proposed an orientation of local binary pattern method for vein recognition. They used Gaussian matched filter to enhance vein images, and then encoded the orientations of local binary patter features, and finally, the Hamming distance was employed for feature matching. Lee [8] proposed a palm vein recognition method based on directional code which is extracted from the response of 2D-Gabor-filtered vein images.

Palm vein recognition method proposed in this paper can be seen as a texture-based one. Considering that the directional information is a distinguishing feature of vein, the method proposed in this paper is based on the directional information derived from a texture descriptor, i.e., the LBP. The flowchart of the proposed method is shown in Fig. 1. Palm vein images are preprocessed to enhance vein patterns. From the enhanced images, the directional features based on LBP are extracted. Angular distances are used to compute distance measurements for recognition. The proposed method employs the directional information of vein patterns, and at the same time takes the advantage of multi-resolution analysis bringing by the LBP. This characteristics of the proposed method makes it get a higher recognition accuracy then the considered literatures shown in the experiments.

In the following part of this paper, we will describe in detail each component of the proposed method with experiments and analysis. The rest of this paper is organized as follows. Section 2 presents the way for deriving directional features from LBP, in which the LBP is firstly reviewed followed by the strategies for deriving directional features. Section 3 provides each of the components of the proposed hand vein recognition method. Experiments with analysis are presented in Section 4 and Section 5 concludes the work. 


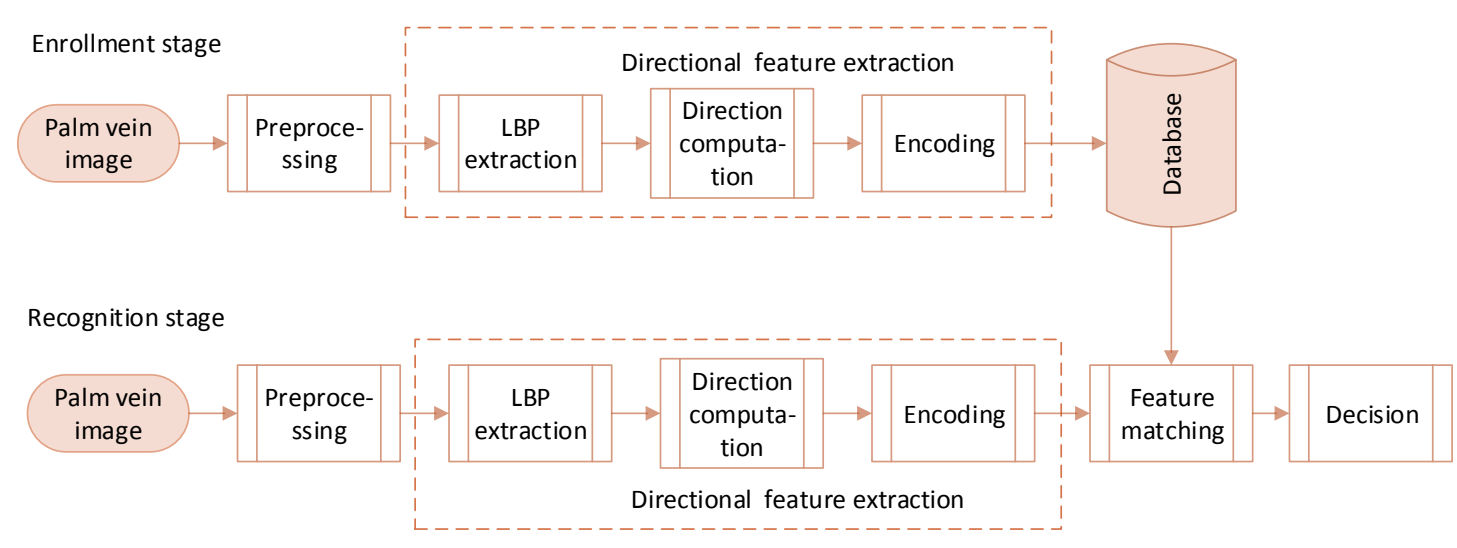

Figure 1. Block Diagram of the Proposed Method

\section{Palm Vein Image Preprocessing}

Palm vein image preprocessing includes region of interest (ROI) extraction and palm vein image enhancement. ROI extraction aims at clipping a most informative region from the whole image, which serves as an image aligning procedure to remove the translation and rotation of images introduced in the data collection, and at the same time to reduce the complexity of the following processing. In this work, the ROI extraction method used in Ref. [3] is employed, and a ROI palm vein image with dimensions of $128 \times 128$ is clipped.

To enhance vein patterns and suppress noises, palm vein images are enhanced by using a multi-scale Gaussian matched filter [18], which contains multi-scale matched filtering and scale production. The multi-scale Gaussian matched filter is expressed as Eq. (1):

$$
\left\{\begin{array}{l}
G(x, y, \theta)=-\exp \left(-\frac{x^{2}}{\sigma_{x}^{2}}\right)-m \\
|x| \leq 3 \sigma_{x} \\
|y| \leq \frac{s L}{2}
\end{array}\right.
$$

where $x=x_{0} \cos \theta+y_{0} \sin \theta, y=-x_{0} \sin \theta+y_{0} \cos \theta,\left(x_{0}, y_{0}\right)$ is the center of the filter, $\theta$ is the direction of the filter, and $\sigma_{x}$ is the standard deviation of the filter along $x$ axis. $m$ is the mean value of the filter, $L$ is the length of the filter in $y$ axis, and $s$ is a scale parameter controlling the filter size. With different values of $\sigma_{x}$ and $L$, filters of different scales can be generated. With these filters, vein patterns with different scales can be enhanced. The filters are used to convolve the palm vein images and the largest response with respect to $\theta$ is taken as the final result, as shown in Eq. (2):

$$
R(x, y)=\max _{\theta}(G(x, y, \theta) * I(x, y))
$$

where $I(x, y)$ is the palm vein image, and $R(x, y)$ represents the convolution response. In this paper, the values of $\theta$ are selected as $\theta=\{0, \pi / 6, \cdots, 5 \pi / 6\}$.

The scale production is defined as the product of filter responses at two adjacent scales, i.e.,

$P_{s_{j}}(x, y)=R_{s_{j}}(x, y) \cdot R_{s_{j+1}}(x, y)$

where $R_{S_{j}}(x, y)$ and $R_{s_{j+1}}(x, y)$ are the responses of two matched filters, whose scales are adjacent. 
Figure 2 shows an example of preprocessed palm vein image, in which Figure 2 (a) is the original palm vein image, Figure 2 (b) is the filtered image under scale 1, Figure 2 (c) is the filtered image under scale 2, and Figure 2 (d) is the final enhanced image.

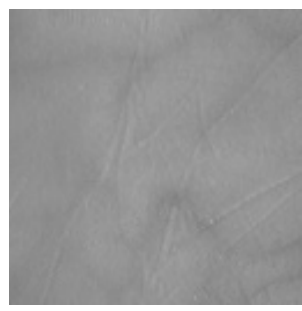

(a) ROI image

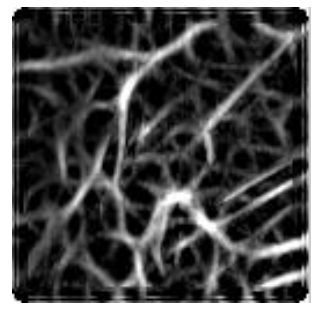

(b) Enhanced image with scale 1

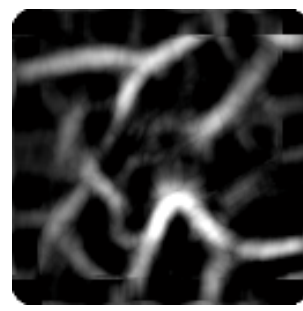

(c) Enhanced image with scale 2

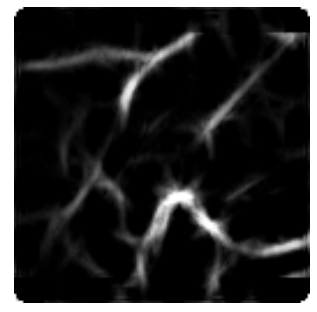

(d) Final enhanced image

Figure 2. Results of Palm Vein Preprocessing

\section{Feature Extraction and Similarity Measurement}

\subsection{A Brief Review of LBP}

The Local Binary Patterns (LBP) [19] is an effective texture descriptor which can characterize the variance of spatial structure of an image patch. Given a central pixel in the image, a real number, which is the LBP value of the pixel, is computed by comparing its intensity value with those of its neighborhoods, as expressed in Eq. (4):

$L B P_{P, R}=\sum_{p=1}^{P} \operatorname{sgn}\left(g_{p}-g_{c}\right) \cdot 2^{P-1}$

where $g_{c}$ is the intensity value of the central pixel, $g_{p}$ is the intensity value of its $p$ th neighboring pixel, $P$ is the number of neighbors, $R$ is the radius of the neighborhood, and $\operatorname{sgn}(\cdot)$ is the sign function, i.e.,

$\operatorname{sgn}(x)= \begin{cases}1, & x \geq 0 \\ 0, & x<0\end{cases}$

If the location of the central pixel is $(0,0)$, the location of its $p$ th neighbor is given by $(-R \cdot \sin (2 \pi / P), R \cdot \cos (2 \pi / P))$. If the locations of the neighboring pixels are not in the center of grids, they can be estimated by interpolation.

Figure 3 shows some examples of neighbor sets for different configurations of $P$ and $R$, in which the neighborhood is circularly symmetric.

\subsection{Deriving Directional Features from LBP}

LBP can effectively describe the local texture patterns of images by computing the local intensity variances. As is known, the direction is an important property of textures, and the direction of LBP can then reflect the directional characteristics of local textures. On the other hand, existing study on vein recognition shows that directional features are effective in representing vein patterns, either in texture-based or structure-based methods. Therefore, we intend to develop a methodology that can extract directional information from LBP descriptors for palm vein feature representation. 

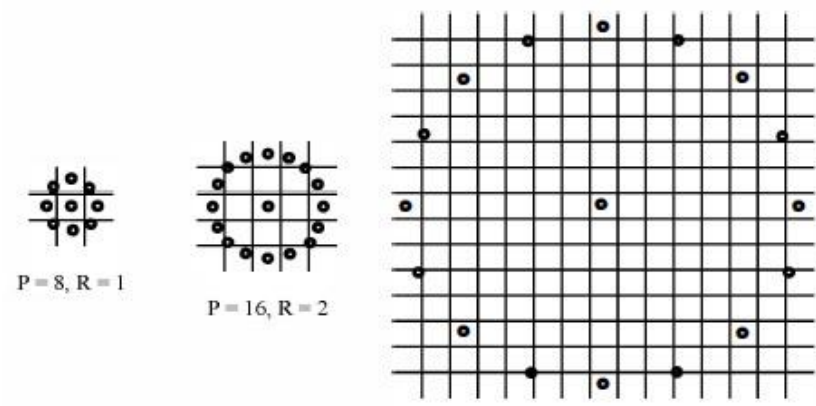

$P=16, R=7$

Figure 3. LBP Neighbor Sets Under Different Configurations of $P$ and $\boldsymbol{R}$

There are many possible ways for extracting directional information from LBP. A typical example is the method proposed by $\mathrm{Bu}$ in Ref. [17], in which the middle position of longest ' 0 ' series in a LBP is computed as its direction, and experiments in their work showed that the direction information extracted from LBP is effective for vein recognition. The reason why the direction information of LBP is effective for vein recognition lies in that in an enhanced vein image, the vein regions have higher intensity values than those of the surroundings, and the value of LBP can reflect whether the local region from which the LBP is computed locates at a vein region, and further, the direction of the vein is reflected by the binary patterns of LBP.

However, in Bu's work, they take only a $3 \times 3$ squared neighborhood for LBP, which causes a loss of multiple scale and multiple resolution characteristics of LBP. As a result, the multi-scale and multi-resolution property of vein patterns cannot be effectively represented.

In this work, we propose a method for deriving directional features from LBP based on $\mathrm{Bu}$ 's conception by exploiting the multiple scale and multiple resolution characteristics of the LBP. The proposed feature extractor can extract more detailed directional features from palm vein images.

To extract directional features from an LBP descriptor, the first step is to define a coordinate system for the descriptor. In this paper, we set up a coordinate system in a LBP descriptor as shown in Figure 4, in which the location of the center pixel of a LBP descriptor is defined as the origin of the coordinate, and the left and right direction are the positive directions of the two axis, respectively.

As was used in Ref. [17], the direction of a LBP descriptor is defined as the middle position of the longest ' 0 ' series in the descriptor, which is formally written as:

direction $=\left[\frac{\text { start }+ \text { end }}{2}\right] \bmod 8$

where start and end are indices of the starting and ending positions of the longest ' 0 ' series in the LBP, and [·] function returns an integer that is no larger than a decimal value. mod is the arithmetical complement operation. 


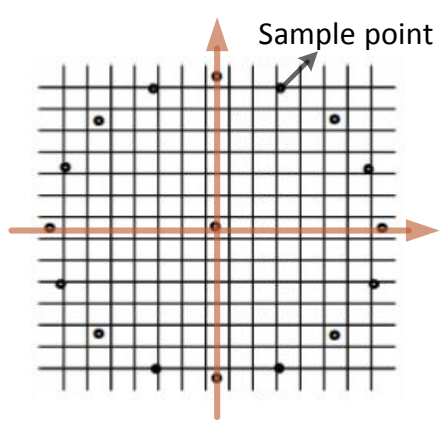

Figure 4. The Setup of the Coordinate System for an LBP Descriptor

Through this way, the descriptor can describe the directional property of vein patterns of different scales. Figure 5 shows some visual examples of the LBP direction with different $P$ and $R$ values, from which it can be seen that larger $P$ value tends to get smoother results, and larger $R$ value can represent vein patterns with larger scales.

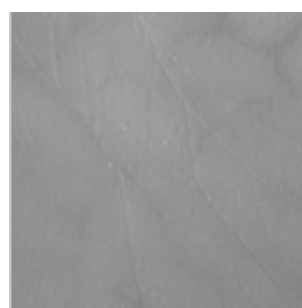

(a) Original image

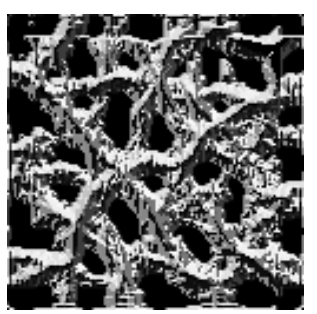

(b) $P=8, R=1$

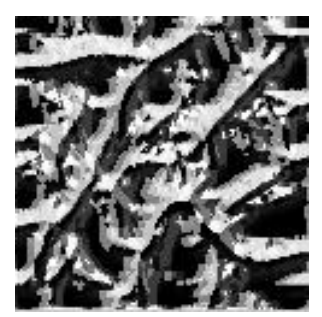

(c) $P=8, R=3$

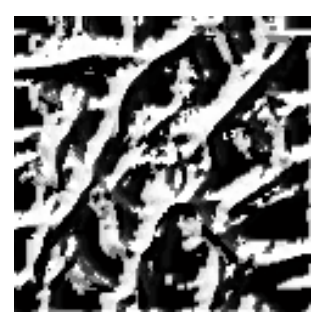

(d) $P=16, R=3$

Figure 5. Some Visual Example of the Direction of LBP

\subsection{Similarity Measurement}

The results of feature extraction using the proposed method is an integer varies in the range $[0, P-1]$, indicating the index of the direction in the coordinate shown in Figure 4. The similarity of such features should reflect the difference between any two directions, and it can be defined as Eq. (7):

$d(x, y)= \begin{cases}\min (p(x, y)-q(x, y), P-(q(x, y)-p(x, y))), & p \geq q \\ \min (q(x, y)-p(x, y), P-(p(x, y)-q(x, y))), & p<q\end{cases}$

where $p(x, y)$ and $q(x, y)$ are the feature value of two palm vein feature maps at location $(x, y)$, respectively, and $P$ is the resolution, i.e., number of neighborhood points in LBP. Eq. (7) indicates that the distance of two directions is defined as the minimal angular between two directions, which is referred as angular distance.

Having the distance measurement of feature value, we can then define the distance between two palm vein feature maps, as is shown in Eq. (8):

$D=\frac{2}{P} \sum_{y=1}^{N} \sum_{x=1}^{M} d(x, y)$

where $M$ and $N$ are the dimensions of the palm vein feature map image, and the coefficient $2 / P$ guarantees that the distance value $D$ ranges in $[0,1]$.

To improve the computational efficiency, the feature value obtained by Eq. (8) can be encoded as binary values, and thus, the distance measurement of two palm vein feature 
maps can be computed by binary operation. According to Ref. [20], the encoding rule can be represented as Eq. (9) (here we restrict that the value of $P$ is even):

$C(x, y, k)= \begin{cases}1, & k \leq W(x, y) \leq k+P / 2 \\ 0, & \text { otherwise }\end{cases}$

Having the encoded representation of the feature value, the similarity measurement can be computed by Eq. (10), which can be effectively implemented by logical operations.

$D=\frac{2}{P} \sum_{y=1}^{N} \sum_{x=1}^{M} \sum_{k=1}^{P / 2} C_{p}(x, y, k) \otimes C_{q}(x, y, k)$

where $C_{p}(x, y, k)$ and $C_{q}(x, y, k)$ are the $k$ th binary values of the encoded feature values of palm vein feature maps $p$ and $q$, respectively, computed using Eq. (9).

\section{Experiments and Discussions}

\subsection{Dataset}

To evaluate the performance of the proposed feature extractor, a most commonly used public dataset is taken into consideration, namely, the PolyU multispectral palmprint database [21] (the NIR spectrum). The PolyU multispectral palmprint images were collected from 250 volunteers. In total, the database contains 6,000 images from 500 different palms for the NIR illumination. Along with the original image, the clipped ROI images are also provided. Figure 6 shows some of the ROI images of the NIR spectrum from the database. For the experiments, half of the images from each palm are randomly selected as the training set for parameter optimization, i.e., 3,000 images for training and 3,000 images for evaluation.
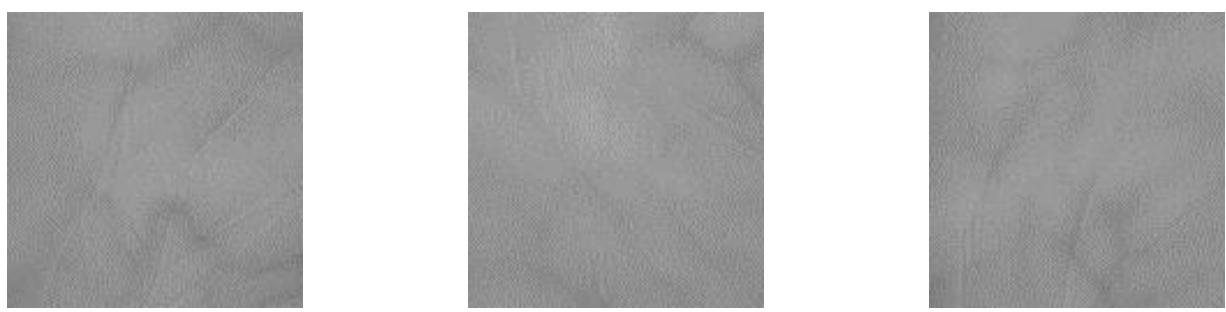

\section{Figure 6. Some Samples of the NIR Spectrum from the PolyU database (ROI Images)}

In this paper, all the experiments are evaluated by the equal error rate (EER). To compute EER, every two samples in the considered database are matched, if the two sample from the same person (class), the matching is counted as a genuine matching, otherwise, an impostor matching. The false acceptance rate (FAR) and the false reject rate (FRR) are then computed using the matching scores, and finally, the point where FAR equals FRR is taken as the EER.

\subsection{Results of Verification}

In this experiment, we intend to evaluate the performance of the proposed method comparing with some of the state-of-the-arts on palm vein recognition. In the experiment, the parameters $P$ and $R$ are set to 16 and 3 whose values are selected by using a crossvalidation scheme. The results are listed in Table 1 and the ROC curves of all the methods are plotted in Figure 7. Along with the results in Table 1, the results of some methods without image enhancement are also reported for comparison, which are listed in brackets. 
Table 1. Verification EER (\%)

\begin{tabular}{ll}
\hline methods & EER \\
\hline \hline Lee [8] & 0.32 \\
\hline Zhang [11] & 0.23 \\
\hline Zhou [7] & 0.004 \\
\hline Bu [17] & $0.040(9.0040)$ \\
\hline Proposed $(R=3, P=16)$ & $0.0017(0.13)$ \\
\hline
\end{tabular}

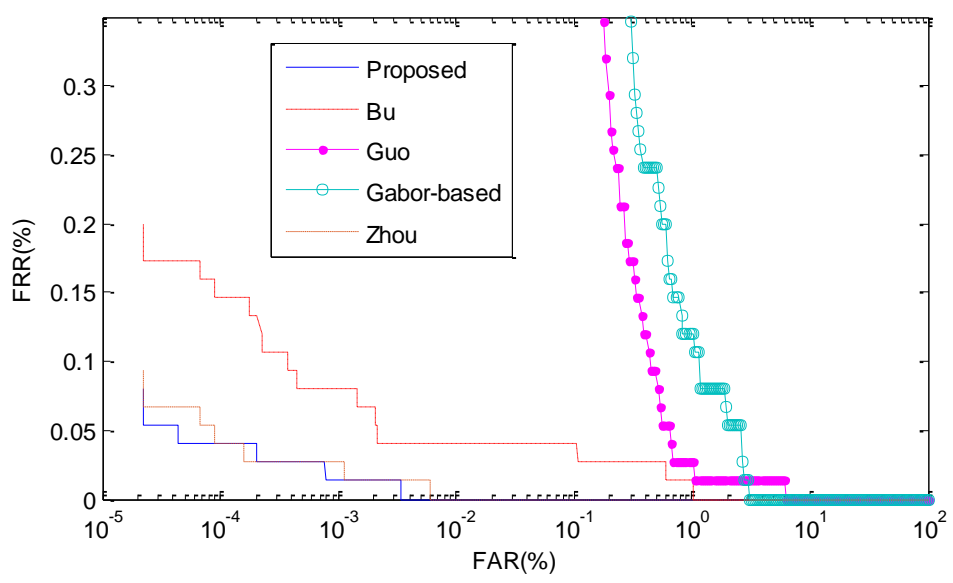

Figure 7. ROC Curves of the Considered Methods (With Enhancement)

From Table 1 and Figure 7, we can see that the proposed method over the considered dataset obtains the highest accuracies compared with the referred literatures, since the proposed method takes into consideration of the directional information of palm vein patterns, which is believed to be the most discriminating features of veins. The method proposed by Lee [8] is a typical texture-based method, which used Gabor filters to extract texture feature from palm vein images. As is explained in this paper, such methods tend to be easily affected by noises. Method proposed by Zhang [11] can be seen as a structurebased method, but they used only the location information of vein patterns, and therefore, other information is loss. Bu's algorithm [17] just used a $3 \times 3$ block neighborhood to compute LBP, and much multiple resolution and scale information are loss, and therefore, the accuracy is lower than the method proposed in this paper. Moreover, it also can be seen from the results that the image enhancement improves the accuracy of the proposed method since vein patterns are enhanced and at the same time background pixels are suppressed by the enhancement.

\subsection{Multi-Scale Analysis}

In the first experiment, the radius and the number of neighborhood of LBP are set to 3 and 16, respectively. As is known, the LBP is essentially multi-resolution, and hence, the proposed method also has the multi-resolution version by setting different values of $P$ and $R$.

It is obvious that different resolutions of the proposed method can gain different performances when applied to palm vein images since the vein patterns are essentially multi-scaled. To decide an optimum values of parameters $P$ and $R$, we perform an exhaustive selection procedure taking the verification EER as the criterion. 
The results of the proposed method with multi-scale are listed in Table 2 and plotted in Figure 8. From the results we can see that the accuracies are apparently affected by the scale and resolution. Generally, the higher the resolution, the higher the accuracies can be obtained. Besides, a larger scale will result in a lower EER, and this can be attributed to the fact that $R=3$ is more close to the scale of the vein patterns in the images.

Table 2. Verification EER of Multi-Scale (\%)

\begin{tabular}{lll}
\hline & $P=8$ & $P=16$ \\
\hline \hline$R=1$ & 0.039 & 0.029 \\
\hline$R=2$ & 0.027 & 0.016 \\
\hline$R=3$ & 0.013 & 0.0017 \\
\hline
\end{tabular}

\subsection{Multimodal Analysis}

Multimodal biometrics is a frequently used technology for enhance the accuracy and security of personal recognition system. The proposed method aims at extracting directional information from texture images, and therefore, theoretically, it is supposed to be effective for feature extraction of other biometric modals.

In this experiment, besides the palm vein images, we apply the proposed algorithm to palmprint images to evaluate its performance over palmprint recognition. Moreover, we also construct a bi-modal biometric system using palm vein and palmprint, since palmprint and palm vein image can be captured at the same time, and therefore, a bimodal biometric system using palmprint and palm vein are the most frequently used in practical systems.

For palmprint images, the proposed method is directly applied to the images, and the parameters (values of $P$ and $R$ ) are also selected over cross-validation. The dataset considered in this experiment is the blue spectrum of the PolyU multispectral palmprint database. The results are listed in Table 3 .

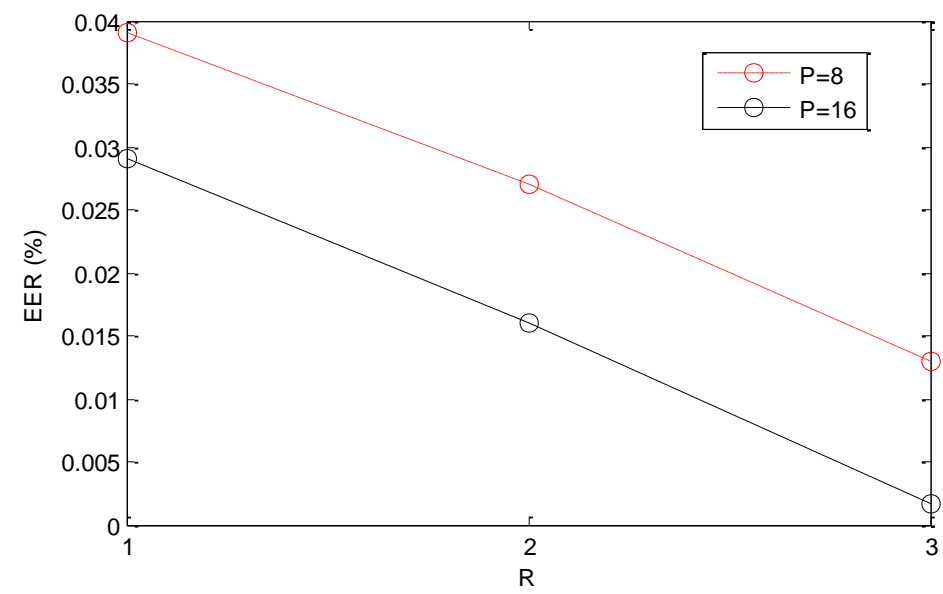

Figure 8. A Figure that Demonstrate Multi-Scale 
Table 3. Verification EER of Palmprint (\%)

\begin{tabular}{lll}
\hline & $P=8$ & $P=16$ \\
\hline \hline$R=1$ & 5.38 & 4.11 \\
\hline$R=2$ & 0.33 & 0.12 \\
\hline$R=3$ & 0.24 & 0.067 \\
\hline
\end{tabular}

From Table 3, we can see that the proposed method is not so effective over palmprint images as those over palm vein images. This is because the directional information extracted in this paper is based on the LBP, which is verity sensitive to the noises. Compared with palm vein images, palmprint textures are finer, and therefore, they are much easier to be affected by noise.

To enhance the verification accuracy, a multimodal biometric system is established using palm vein and palmprint images. The score level fusion is used form multiple modal fusion as shown in Eq. (11):

$D_{\text {fusion }}=\omega D_{\text {vein }}+(1-\omega) D_{\text {palmprint }}$

where $D_{\text {vein }}$ and $D_{\text {palmprint }}$ are the distance measurement of palm vein and palmprint, respectively. $\omega$ is the weight whose value is decided by cross-validation on the training dataset.

The results of multimodal system is further improved as $0.0012 \%$ EER. We can see that after fusion, the verification accuracy of the proposed method are further enhanced, which is higher than either single modal of palmprint and palm vein.

\section{Conclusions}

In this paper, a palm vein recognition method based on directional information derived from local binary patterns (LBP) is proposed. Experiments are conducted on a publicly available database. The results show that the proposed method can effectively represent palm vein features and therefore obtain a high verification accuracy. This can be attributed to the fact that the directional information, which is the most discriminative information of vein patterns is employed. Besides, we show that the proposed method can also be used in palmprint recognition. Due to its effectiveness in representing both palm vein and palmprint features, the proposed method can be employed in bi-modal biometric systems, which can obtain a further higher accuracy than any single modal. Moreover, the distance measurement computation is implemented using binary operation, the efficiency of the proposed method can be guaranteed, and therefore it can be employed in online biometric systems.

\section{Acknowledgement}

This work was supported by the Harbin University Youth Scientific Research Foundation under Grant No. HUYF2013-024, Harbin University Student Scientific Research Foundation under Grant No. HXS2013065, and Harbin University Doctoral Scientific Research Foundation under Grant No. HUDF2014-008.

\section{References}

[1] R. Sharma, N. Mishra and S. K. Yadav, "Fingerprint Recognition System and Techniques: A Survey", International Journal of Scientific \& Engineering Research, vol. 4, no. 6, (2013), pp. 1670.

[2] H. Hasan and S. Abdul-Kareem, "Fingerprint image enhancement and recognition algorithms: a survey", Neural Computing and Applications, vol. 23, no. 6, (2013), pp. 1605-1610.

[3] D. Zhang, K. Wai-Kin, J. You and M. Wong, "Online palmprint identification. Pattern Analysis and Machine Intelligence", IEEE Transactions on vol. 25, no. 9, (2003), pp. 1041-1050. 
[4] D. Zhang, W. Zuo and F. Yue, "A Comparative Study of Palmprint Recognition Algorithms", ACM COMPUTING SURVEYS, vol. 44, no. 1, (2012), pp. 159-170.

[5] N. Duta, "A survey of biometric technology based on hand shape", Pattern Recognition, vol. 42, no. 11, (2009), pp. 2797-2806, 11.

[6] S. Sharma, S. R. Dubey, S. K. Singh, R. Saxena and R. K. Singh, "Identity verification using shape and geometry of human hands", Expert Systems with Applications, vol. 42, no. 2, (2015), pp. 821-832.

[7] Z. Yingbo and A. Kumar, "Human Identification Using Palm-Vein Images", Information Forensics and Security, IEEE Transactions on, vol. 6, no. 4, (2011), pp. 1259-1274.

[8] J.-C. Lee, "A novel biometric system based on palm vein image. Pattern Recognition Letters, vol. 33, no. 12, (2012), pp. 1520-1528.

[9] K.-S. Wu, J.-C. Lee, T.-M. Lo, K.-C. Chang and C.-P. Chang, "A secure palm vein recognition system", Journal of Systems and Software, vol. 86, no. 11, (2013), pp. 2870-2876.

[10] A. Kumar and Z. Yingbo, "Human Identification Using Finger Images", Image Processing, IEEE Transactions on, vol. 21, no. 4, (2012), pp. 2228-2244.

[11] D. Zhang, Z. Guo, G. Lu, L. Zhang, Y. Liu and W. Zuo, "Online joint palmprint and palmvein verification", Expert Systems with Applications, vol. 38, no. 3, (2011), pp. 2621-2631.

[12] B. Huang, Y. Dai, R. Li, D. Tang and W. Li, "Finger-vein authentication based on wide line detector and pattern normalization", pp. 1269-1272.

[13] L. Wang, G. Leedham and D. S.-Y. Cho, "Minutiae feature analysis for infrared hand vein pattern biometrics", Pattern recognition, vol. 41, no. 3, (2008), pp. 920-929.

[14] N. Miura, A. Nagasaka and T. Miyatake, "Feature extraction of finger-vein patterns based on repeated line tracking and its application to personal identification", Machine Vision and Applications, vol. 15, no. 4, (2004), pp. 194-203.

[15] W.-Y. Han and J.-C. Lee, "Palm vein recognition using adaptive Gabor filter", Expert Systems with Applications, vol. 39, no. 18, (2012), pp. 13225-13234.

[16] J. Yang and X. Li, "Efficient Finger Vein Localization and Recognition", Pattern Recognition (ICPR), 2010 20th International Conference on, (2010), pp. 1148-1151.

[17] W. Bu, X. Wu and E. Gao, "Hand vein recognition based on orientation of LBP. Sensing Technologies for Global Health, Military Medicine, Disaster Response, and Environmental Monitoring II; and Biometric Technology for Human Identification", IX, vol. 8371, no. 6, (2012), pp. 561-564.

[18] Y.-B. Zhang, Q. Li, J. You and P. Bhattacharya, "Palm vein extraction and matching for personal authentication", Advances in Visual Information Systems, Springer, pp. 154-164 (2007).

[19] T. Ojala, M. Pietikainen and T. Maenpaa, "Multiresolution gray-scale and rotation invariant texture classification with local binary patterns", Pattern Analysis and Machine Intelligence, IEEE Transactions on, vol. 24, no. 7, (2002), pp. 971-987.

[20] Z. Wangmeng, L. Zhouchen, G. Zhenhua and D. Zhang, "The multiscale competitive code via sparse representation for palmprint verification", pp. 2265-2272.

[21] PolyU multispectral palmprint Database. http://www.comp.polyu.edu.hk/ biometrics/ MultispectralPalmprint/MSP.htm 
International Journal of Signal Processing, Image Processing and Pattern Recognition Vol. 9, No. 5 (2016) 